\title{
Applications of Modified Sigmoid Functions to a Class of Starlike Functions
}

\author{
Muhammad Ghaffar Khan, ${ }^{1}$ Bakhtiar Ahmad ${ }^{1}{ }^{2}$ \\ Gangadharan Murugusundaramoorthy $\mathbb{D}^{3},{ }^{3}$ Ronnason Chinram $\mathbb{D}^{4},{ }^{4}$ \\ and Wali Khan Mashwani ${ }^{1}{ }^{1}$ \\ ${ }^{1}$ Institute of Numerical Sciences, Kohat University of Science and Technology, Kohat, Pakistan \\ ${ }^{2}$ Government Degree College Mardan, 23200 Mardan, Pakistan \\ ${ }^{3}$ Department of Mathematics, SAS, Vellore Institute of Technology, Vellore 632014, India \\ ${ }^{4}$ Algebra and Applications Research Unit, Division of Computational Science, Faculty of Science, Prince of Songkla University, \\ Hat Yai, Songkhla 90110, Thailand
}

Correspondence should be addressed to Ronnason Chinram; ronnason.c@psu.ac.th

Received 17 September 2020; Revised 11 October 2020; Accepted 1 November 2020; Published 19 November 2020

Academic Editor: Acu Ana Maria

Copyright (C) 2020 Muhammad Ghaffar Khan et al. This is an open access article distributed under the Creative Commons Attribution License, which permits unrestricted use, distribution, and reproduction in any medium, provided the original work is properly cited.

The main focus of this investigation is the applications of modified sigmoid functions. Due to its various uses in physics, engineering, and computer science, we discuss several geometric properties like necessary and sufficient conditions in the form of convolutions for functions to be in the special class $\mathcal{S}_{S G}^{*}$ earlier introduced by Goel and Kumar and obtaining third-order Hankel determinant for this class using modified sigmoid functions. Also, the third-order Hankel determinant for 2- and 3-fold symmetric functions of this class is evaluated.

\section{Introduction}

In this section, we present the related material for better understanding of the concepts discussed later in this article. We start with the notation of $\mathscr{A}$, the class of functions $f$ which are analytic in $\mathbb{U}=\{z \in \mathbb{C}:|z|<1\}$ and its series representation is

$$
f(z)=z+\sum_{n=2}^{\infty} a_{n} z^{n}, z \in \mathbb{U} .
$$

Further, a subclass of class $\mathscr{A}$ which is denoted by $\delta$ contains all univalent functions in $\mathbb{U}$. Bieberbach conjectured in 1916 that $\left|a_{n}\right| \leq n, n=2,3, \cdots$. De Branges proved this in 1985; see [1]. During this period, a lot of coefficient results were established for some subfamilies of $\delta$. Some of these classes are the class $\mathcal{S}^{*}$, known as the class of starlike functions, the class $\mathscr{K}$, known as class of convex functions, and $\mathscr{R}$ of bounded turning functions. These are defined as

$$
\begin{aligned}
& \mathcal{S}^{*}(\psi)=\left\{f \in \mathcal{S}: \frac{z f^{\prime}(z)}{f(z)}<\psi=\frac{1+z}{1-z}, z \in \mathbb{U}\right\}, \\
& \mathscr{K}(\psi)=\left\{f \in \mathcal{S}: \frac{\left(z f^{\prime}(z)\right)^{\prime}}{f^{\prime}(z)}<\psi=\frac{1+z}{1-z}, z \in \mathbb{U}\right\}, \\
& \mathscr{R}(\psi)=\left\{f \in \mathcal{S}: f^{\prime}(z) \prec \psi=\frac{1+z}{1-z}, z \in \mathbb{U}\right\} .
\end{aligned}
$$

Now, recall the subordination definition; we say that an analytic function $f_{1}(z)$ is subordinate to $f_{2}(z)$ in $\mathbb{U}$ and is symbolically written as $f_{1}(z) \prec f_{2}(z)$ if there occurs a Schwarz function $u(z)$ with properties that $|u(z)| \leq 1$ and $u(0)=1$ such that $f_{1}(z)=f_{2}(u(z))$. Moreover, if $f_{2}(z)$ is in the class $\mathcal{S}$, then we have the following equivalency, due to $[2,3]$, 


$$
\begin{aligned}
& f_{1}(0)=f_{2}(0), \\
& f_{1}(\mathbb{U}) \subseteq f_{2}(\mathbb{U}) .
\end{aligned}
$$

For two functions $f_{1}(z)=z+\sum_{n=2}^{\infty} a_{n, 1} z^{n}$ and $f_{2}(z)=z$ $+\sum_{n=2}^{\infty} a_{n, 2} z^{n}$ in $\mathbb{U}$, then the convolution or Hadamard product is defined by

$$
\left(f_{1} * f_{2}\right)(z)=z+\sum_{n=2}^{\infty} a_{n, 1} a_{n, 2} z^{n} .
$$

By varying the right-hand side of subordinated inequality in (2), several familiar classes can be obtained such as the following:

(1) For $\psi=(1+A z) /(1+B z)$, we get the class $\mathcal{S}^{*}(A, B)$; see [4] for details

(2) While for different values of $A$ and $B$ the class $\mathcal{S}^{*}(\alpha)$ $=\mathcal{S}^{*}(1-2 \alpha,-1)$ is obtained and investigated in [5]

(3) For $\psi=1+\left(2 / \pi^{2}\right)(\log ((1+\sqrt{z}) /(1-\sqrt{z})))^{2}$, the class was defined and studied in [6]

(4) For $\psi=\sqrt{1+z}$, the class is denoted by $\mathcal{S}_{L}^{*}$; details can be seen in $[7,8]$, and for further study, see [9]

(5) For $\psi=\cosh (z)$, the class is denoted by $\mathcal{S}_{\text {cosh }}^{*}$; see [10]

(6) For $\psi=1+\sin (z)$, the class is denoted by $\mathcal{S}_{\text {sin }}^{*}$; see [11] for details, and for further investigation, see [12]

(7) While for $\psi=z+\sqrt{1+z^{2}}$, the class is denoted by $\mathcal{S}_{l}^{*}$; see [13]

(8) For $\psi=e^{z}$, the class denoted by $\mathcal{S}_{e}^{*}$ was defined and studied in $[14,15]$

(9) Similarly, if $\psi=1+(4 / 3) z+(2 / 3) z^{2}$, then such a class is denoted by $\mathcal{S}_{C}^{*}$ and was introduced in [16], and for further study, the reader is referred to [17]

Also, several other subclasses of starlike functions were introduced recently in [18-22] by choosing some particular function for $\psi$ such functions are associated with Bell numbers, shell-like curve connected with Fibonacci numbers, and functions connected with the conic domains.

In this paper, we investigate starlike functions associated with a kind of special functions known as modified sigmoid function $\psi(z)=2 /\left(1+e^{-z}\right)$. In mathematics, the theory of special functions is the most important for scientists and engineers who are concerned with actual mathematical calculations. To be specific, it has applications in problems of physics, engineering, and computer science. The activation function is an example of special function. These functions act as a squashing function which is the output of a neuron in a neural network between certain values (usually 0 and 1 and -1 and 1). There are three types of functions, namely, piecewise linear function, threshold function, and sigmoid function. In the hardware implementation of neural network, the most important and popular activation function is the sigmoid function. The sigmoid function is often used with gradient descendent type learning algorithm. Due to differentiability of the sigmoid function, it is useful in weightlearning algorithm. The sigmoid function increases the size of the hypothesis space that the network can represent. Some of its advantages are the following:

(1) It gives real numbers between 0 and 1

(2) It maps a very large output domain to a small range of outputs

(3) It never loses information because it is a one-to-one function

(4) It increases monotonically

For more details, see [23].

The class $\mathcal{S}_{S G}^{*}$ defined by Goel and Kumar in [24] is defined as

$$
\frac{z f^{\prime}(z)}{f(z)} \prec \frac{2}{1+e^{-z}},(z \in \mathbb{U}) .
$$

For a parameter $q$, with $n \in \mathbb{N}=\{1,2,3, \cdots\}$, Pommerenke $[25,26]$ defined Hankel determinant $H_{q, n}(f)$ for functions $f \in \mathcal{S}$ of the form (1) as follows:

$$
H_{q, n}(f)=\left|\begin{array}{cccc}
a_{n} & a_{n+1} & \cdots & a_{n+q-1} \\
a_{n+1} & a_{n+2} & \cdots & a_{n+q} \\
\vdots & \vdots & \cdots & \vdots \\
a_{n+q-1} & a_{n+q} & \cdots & a_{n+2 q-2}
\end{array}\right| .
$$

The growth of $H_{q, n}(f)$ has been evaluated for different subcollections of univalent functions. Exceptionally, for each of the sets $\mathscr{K}, \mathcal{S}^{*}$, and $\mathscr{R}$, the sharp bound of the determinant $H_{2,2}(f)=\left|a_{2} a_{4}-a_{3}^{2}\right|$ was found by Jangteng et al. [7, 27], while for the family of close-to-convex functions the sharp estimate is still unknown (see [28]). On the other hand, for the set of Bazilevic functions, the best estimate of $\left|H_{2,2}(f)\right|$ was proved by Krishna et al. [29]. For more work on $H_{2,2}(f)$, see [30-34].

The determinant

$$
H_{3,1}(f)=\left|\begin{array}{ccc}
1 & a_{2} & a_{3} \\
a_{2} & a_{3} & a_{4} \\
a_{3} & a_{4} & a_{5}
\end{array}\right|
$$

is known as the third-order Hankel determinant, and the estimation of this determinant $\left|H_{3,1}(f)\right|$ is the focus of various researchers of this field. In 2010, the first article on $H_{3,1}$ $(f)$ was published by Babalola [35], in which he obtained the upper bound of $\left|H_{3,1}(f)\right|$ for the classes of $\mathcal{S}^{*}, \mathscr{K}$, and $\mathscr{R}$. Later on, a few mathematicians extended this work for various subcollections of holomorphic and univalent 
functions; see [36-41]. In 2017, Zaprawa [42] improved their work by proving

$$
\left|H_{3,1}(f)\right| \leq \begin{cases}1, & \text { for } f \in \mathcal{S}^{*}, \\ \frac{49}{540}, & \text { for } f \in \mathscr{K}, \\ \frac{41}{60}, & \text { for } f \in \mathscr{R} .\end{cases}
$$

And he asserted that these inequalities are not sharp as well. Additionally, for the sharpness, he investigated the subfamilies of $\mathcal{S}^{*}, \mathscr{C}$, and $\mathscr{R}$ comprising functions with $m$-fold symmetry and acquired the sharp bounds. Recently, in 2018, Kowalczyk et al. [43] and Lecko et al. [44] got the sharp inequalities which are

$$
\begin{aligned}
& \left|H_{3,1}(f)\right| \leq 4 / 135, \\
& \left|H_{3,1}(f)\right| \leq 1 / 9,
\end{aligned}
$$

for the classes $\mathscr{K}$ and $\mathcal{S}^{*}(1 / 2)$, respectively, where the symbol $\mathcal{S}^{*}(1 / 2)$ indicates the family of starlike functions of order $1 / 2$. Additionally, in 2018, the authors [45] got an improved bound $\left|H_{3,1}(f)\right| \leq 8 / 9$ for $f \in \mathcal{S}^{*}$, which is yet not the best possible. In this article, our main purpose is to study necessary and sufficient conditions for functions to be in the class $\mathcal{S}_{S G}^{*}$ in the form of convolutions results, coefficient inequality, and important third-order Hankel determinant for this class in (7) and also for its 2- and 3-fold symmetric functions.

\section{A Set of Lemmas}

Let $\mathscr{P}$ be the family of functions $p(z)$ that are holomorphic in $\mathbb{D}$ with $\mathfrak{R e} p(z)>0$ and its series form is as follows:

$$
p(z)=1+\sum_{n=1}^{\infty} c_{n} z^{n}(z \in \mathbb{D}) .
$$

Lemma 1. If $p(z) \in \mathscr{P}$ and it is of the form (12), then

$$
\begin{aligned}
\left|c_{n}\right| & \leq 2 \text { for } n \geq 1, \\
\left|c_{n+k}-\delta c_{n} c_{k}\right| & \leq 2 \text { for } 0 \leq \delta \leq 1, \\
\left|c_{n} c_{m}-c_{l} c_{k}\right| & \leq 4 \text { for } n+m=l+k, \\
\left|c_{n+2 k}-\delta c_{n} c_{k}^{2}\right| & \leq 2(1+2 \delta) \text { for } \delta \in \mathbb{R}, \\
\left|c_{2}-\frac{c_{l}^{2}}{2}\right| & \leq 2-\frac{\left|c_{1}\right|^{2}}{2},
\end{aligned}
$$

and for $\xi \in \mathbb{C}$.

$$
\left|c_{2}-\xi c_{1}^{2}\right| \leq 2 \max \{1 ;|2 \xi-1|\} .
$$

For the results in (13), (14), (15), (16), and (17), see [46]. Also, see [47] for (18).

Lemma 2. [48]. If $p(z) \in \mathscr{P}$ and is represented by (12), then

$$
\left|c_{2}-v c_{1}^{2}\right| \leq \begin{cases}-4 v+2 & (v \leq 0) \\ 2 & (0 \leq v \leq 1) \\ 4 v-2 & (v \geq 1)\end{cases}
$$

Lemma 3. Let $p \in \mathscr{P}$ have representation of the form (12), then

$$
\left|\alpha c_{1}^{3}-\beta c_{1} c_{2}+\gamma c_{3}\right| \leq 2|\alpha|+2|\beta-2 \alpha|+2|\alpha-\beta+\gamma| .
$$

Proof. Consider the left-hand side of (20) and then rearranging the terms, we have

$$
\begin{aligned}
\left|\alpha c_{1}^{3}-\beta c_{1} c_{2}+\gamma c_{3}\right|= & \mid \alpha\left(c_{1}^{3}-2 c_{1} c_{2}+c_{3}\right)-(\beta-2 \alpha)\left(c_{1} c_{2}-c_{3}\right) \\
& +(\alpha-\beta+\gamma) c_{3}|\leq| \alpha|| c_{1}^{3}-2 c_{1} c_{2}+c_{3} \mid \\
& +|\beta-2 \alpha|\left|c_{1} c_{2}-c_{3}\right|+|\alpha-\beta+\gamma|\left|c_{3}\right| \\
\leq & 2|\alpha|+2|\beta-2 \alpha|+2|\alpha-\beta+\gamma|,
\end{aligned}
$$

where we have used (13) and (14).

\section{Convolution Results for Class $\mathcal{S}_{S G}^{*}$}

Theorem 4. Let $f(z) \in \mathscr{A}$ be the form (1), then $f(z) \in \mathcal{S}_{S G}^{*}$, if and only if

$$
\frac{1}{z}\left[f(z) * \frac{z-\alpha z^{2}}{(1-z)^{2}}\right] \neq 0,
$$

for all $\alpha=\alpha_{\xi}=2 /\left(1-e^{-\xi}\right)$ and also for $\alpha=1$.

Proof. Since $f(z) \in \mathcal{S}_{S G}^{*}$ is analytic in domain $\mathbb{U}$, so $f(z) \neq 0$, for all $z \in \mathbb{U}^{*}=\mathbb{U}\{0\}$, that is $(1 / z) f(z) \neq 0$ for $z \in \mathbb{U}$, which is equivalent to (22) for $\alpha=1$. In this case, the proof is completed. Now, from definition (7), there occurs a Schwarz function $u(z)$, such that $|u(z)|<1$ and $u(0)=0$, such that

$$
\frac{z f^{\prime}(z)}{f(z)}=\frac{2}{1+e^{-u(z)}} .
$$

Equivalently,

$$
\frac{z f^{\prime}(z)}{f(z)} \neq \frac{2}{1+e^{-\xi}}, \quad|\xi|=1
$$

which implies that

$$
z f^{\prime}(z)-f(z) \frac{2}{1+e^{-\xi}} \neq 0
$$


We know that

$$
\begin{aligned}
z f^{\prime}(z) & =f(z) * \frac{z}{(1-z)^{2}} \\
f(z) & =f(z) * \frac{z}{1-z}
\end{aligned}
$$

By simple computation, equation (25) becomes

$$
\frac{1}{z}\left[f(z) * \frac{z-\alpha z^{2}}{(1-z)^{2}}\right] \neq 0
$$

where $\alpha$ is given above.

Conversely, suppose equation (22) holds true for $\alpha=1$, it implies that $(1 / z) f(z) \neq 0$, for all $z \in \mathbb{U}$. Let $\Phi(z)=z f^{\prime}(z) / f(z)$ be analytic in $\mathbb{U}$, with $\Phi(0)=1$. Also, suppose that $\Psi(z)=2 /\left(1+e^{-z}\right), z \in \mathbb{U}$. It is clear from (24) that $\Psi(\partial \mathbb{U}) \cap \Phi(\mathbb{U})=\phi$. Hence, the simply connected domain $\Phi(\mathbb{U})$ is contained in connected component of $\Psi$ $(\partial \mathbb{U})$. The univalence of " $\Psi$ ", together with the fact $\Phi(0)=\Psi(0)=1$, shows that $\Phi \prec \Psi$ and implies that $f(z) \in \mathcal{S}_{S G}^{*}$.

Theorem 5. Let $f(z) \in \mathscr{A}$ be of the form (1), then the necessary and sufficient condition for function $f(z)$ that belongs to class $\mathcal{S}_{S G}^{*}$ is

$$
1-\sum_{n=2}^{\infty}\left(\frac{n\left(1-e^{-\xi}\right)-2}{1-e^{-\xi}}\right) a_{n} z^{n-1} \neq 0 .
$$

Proof. In the light of Theorem 4, we show that $\mathcal{S}_{S G}^{*}$ if and only if

$$
\begin{aligned}
0 \neq \frac{1}{z}\left[f(z) * \frac{z-\alpha z^{2}}{(1-z)^{2}}\right] & =\frac{1}{z}\left[z f^{\prime}(z)-\alpha\left(z f^{\prime}(z)-f(z)\right)\right] \\
& =1-\sum_{n=2}^{\infty}((\alpha-1) n-\alpha) a_{n} z^{n-1} \\
& =1-\sum_{n=2}^{\infty}\left(\frac{n\left(1-e^{-\xi}\right)-2}{1-e^{-\xi}}\right) a_{n} z^{n-1}
\end{aligned}
$$

Hence, the proof is completed.

Theorem 6. Let $f \in \mathscr{A}$ be of the form (1) and satisfies

$$
\sum_{n=2}^{\infty}\left|\frac{n\left(1-e^{-\xi}\right)-2}{1-e^{-\xi}}\right|\left|a_{n}\right|<1,
$$

then $f \in \mathcal{S}_{S G}^{*}$.
Proof. To show $f \in \mathcal{S}_{S G}^{*}$, we have to show that (28) is satisfied. Consider

$$
\begin{aligned}
\left|1-\sum_{n=2}^{\infty}((\alpha-1) n-\alpha) a_{n} z^{n-1}\right| & >1-\sum_{n=2}^{\infty}\left|((\alpha-1) n-\alpha) a_{n} z^{n-1}\right| \\
& =1-\sum_{n=2}^{\infty}|((\alpha-1) n-\alpha)|\left|a_{n}\right||z|^{k-1} \\
& >1-\sum_{n=2}^{\infty}|((\alpha-1) n-\alpha)|\left|a_{n}\right| \\
& =1-\sum_{n=2}^{\infty}\left|\frac{n\left(1-e^{-\xi}\right)-2}{1-e^{-\xi}}\right|\left|a_{n}\right|>0,
\end{aligned}
$$

so by Theorem $5, f(z) \in \mathcal{S}_{S G}^{*}$.

\section{Upper Bound $H_{3,1}(f)$ for Set $\delta_{S G}^{*}$}

Theorem 7. Let $f \in \mathcal{S}_{S G}^{*}$ and is of the form (1), then

$$
\left|a_{3}-\lambda a_{2}^{2}\right| \leq \frac{1}{4} \max \left\{1, \frac{|2 \lambda-1|}{2}\right\}
$$

Proof. Since $f \in \mathcal{S}_{S G}^{*}$, then there exists an analytic function $w(z),|w(z)| \leq 1$ and $w(0)=0$, such that

$$
\frac{z f^{\prime}(z)}{f(z)}=\frac{2}{1+e^{-w(z)}} .
$$

Denote

$$
\begin{aligned}
\Psi(w(z)) & =\frac{2}{1+e^{-w(z)}}, \\
k(z) & =1+c_{1} z+c_{2} z^{2}+\cdots=\frac{1+w(z)}{1-w(z)} .
\end{aligned}
$$

Obviously, the function $k(z) \in \mathscr{P}$ and $w(z)=(k(z)-1) /$ $(k(z)+1)$. This gives

$$
w(z)=\frac{k(z)-1}{k(z)+1}=\frac{c_{1} z+c_{2} z^{2}+c_{3} z^{3}+\cdots}{2+c_{1} z+c_{2} z^{2}+c_{3} z^{3}+\cdots}
$$

$$
\begin{aligned}
\frac{2}{1+e^{-w(z)}}= & +\frac{1}{4} c_{1} z+\left(\frac{1}{4} c_{2}-\frac{1}{8} c_{1}^{2}\right) z^{2} \\
& +\left(\frac{11}{192} c_{1}^{3}-\frac{1}{4} c_{2} c_{1}+\frac{1}{4} c_{3}\right) z^{3} \\
& +\left(\frac{1}{4} c_{1}^{2} c_{2}-\frac{1}{2} c_{3} c_{1}-\frac{1}{4} c_{2}^{2}+\frac{1}{2} c_{4}\right) z^{4}+\cdots
\end{aligned}
$$


while

$$
\begin{aligned}
\frac{z f^{\prime}(z)}{f(z)} & =1+a_{2} z+\left(2 a_{3}-a_{2}^{2}\right) z^{2}+\left(a_{2}^{3}-3 a_{2} a_{3}+3 a_{4}\right) z^{3} \\
& +\left(-a_{2}^{4}+4 a_{2}^{2} a_{3}-4 a_{2} a_{4}-2 a_{3}^{2}+4 a_{5}\right) z^{4}+\cdots
\end{aligned}
$$

On equating coefficients of (36) and (37), we get

$$
\begin{aligned}
& a_{2}=\frac{1}{4} c_{1}, \\
& a_{3}=\frac{1}{8} c_{2}-\frac{1}{32} c_{1}^{2}, \\
& a_{4}=\frac{7}{1152} c_{1}^{3}-\frac{5}{96} c_{2} c_{1}+\frac{1}{12} c_{3}, \\
& a_{5}=-\frac{1}{16}\left(\frac{17}{1152} c_{1}^{4}-\frac{7}{24} c_{1}^{2} c_{2}+\frac{2}{3} c_{3} c_{1}+\frac{3}{8} c_{2}^{2}-c_{4}\right) .
\end{aligned}
$$

Now from (38) and (39), we have

$$
\left|a_{3}-\lambda a_{2}^{2}\right|=\frac{1}{8}\left|c_{2}-\frac{2 \lambda+1}{4} c_{1}^{2}\right| .
$$

Now, using (18), we get the required result.

If we put $\lambda=1$, the above result becomes as follows.

Corollary 8. Let $f(z) \in \mathcal{S}_{S G}^{*}$ be of the form (1) then

$$
\left|a_{3}-a_{2}^{2}\right| \leq \frac{1}{4}
$$

The result is best possible for function

$$
f(z)=z \exp \left(\int_{0}^{z} \frac{e^{t^{2}}-1}{t\left(e^{t^{2}}+1\right)} d t\right)=z+\frac{1}{4} z^{3}+\cdots
$$

Theorem 9. Let $f(z) \in \mathcal{S}_{S G}^{*}$ be of the form (1), then

$$
\left|a_{2} a_{3}-a_{4}\right| \leq \frac{1}{6} .
$$

The result is best possible for function defined as

$$
f_{n}(z)=z \exp \left(\int_{0}^{z} \frac{e^{t^{3}}-1}{t\left(e^{t^{3}}+1\right)} d t\right)=z+\frac{1}{6} z^{4}+\cdots
$$

Applying Lemma 3, we get the required result.

Proof. By using (38), (39), and (40), we get

$$
\left|a_{2} a_{3}-a_{4}\right|=\left|\frac{1}{72} c_{1}^{3}-\frac{1}{12} c_{2} c_{1}+\frac{1}{12} c_{3}\right| .
$$

Applying Lemma 3, we get the required result.
Theorem 10. Let $f(z) \in \mathcal{S}_{S G}^{*}$ be of the form, (1) then

$$
\left|a_{2} a_{4}-a_{3}^{2}\right| \leq \frac{55}{576} .
$$

Proof. With the help of (38), (39), and (40), we get

$$
\left|a_{2} a_{4}-a_{3}^{2}\right|=\left|\frac{1}{48} c_{3} c_{1}-\frac{7}{9216} c_{1}^{4}-\frac{1}{192} c_{1}^{2} c_{2}-\frac{1}{64} c_{2}^{2}\right| .
$$

Now, rearranging the terms

$$
\left|a_{2} a_{4}-a_{3}^{2}\right|=\left|\frac{c_{1}}{192}\left(c_{3}-c_{1} c_{2}\right)-\frac{c_{1} c_{3}-c_{2}^{2}}{64}-\frac{7}{9216} c_{1}^{4}\right| .
$$

Using (13), (14), and (15), we get the required result.

For the third Hankel determinant, we need the following result.

Lemma 11. [24]. Let $f(z) \in \mathcal{S}_{S G}^{*}$ be of the form (1). Then,

$$
\begin{aligned}
& \left|a_{2}\right| \leq \frac{1}{2}, \\
& \left|a_{3}\right| \leq \frac{1}{4}, \\
& \left|a_{4}\right| \leq \frac{1}{6}, \\
& \left|a_{5}\right| \leq \frac{1}{8} .
\end{aligned}
$$

These results are sharp for function defined as

$$
f_{n}(z)=z \exp \left(\int_{0}^{z} \frac{e^{t^{n-1}}-1}{t\left(e^{t^{n-1}}+1\right)} d t\right), \quad \text { for } a_{n}(n=2,3,4,5) \text {. }
$$

Theorem 12. Let $f(z) \in \mathcal{S}_{S G}^{*}$ be of the form (1). Then,

$$
\left|H_{3,1}(f)\right| \leq \frac{191}{2304} \simeq 0.0829 \text {. }
$$

Proof. Since

$$
H_{3,1}(f)=a_{3}\left(a_{2} a_{4}-a_{3}^{2}\right)-a_{4}\left(a_{4}-a_{2} a_{3}\right)+a_{5}\left(a_{3}-a_{2}^{2}\right),
$$

by applying triangle inequality, we obtain

$$
\left|H_{3,1}(f)\right| \leq\left|a_{3}\right|\left|a_{2} a_{4}-a_{3}^{2}\right|+\left|a_{4}\right|\left|a_{4}-a_{2} a_{3}\right|+\left|a_{5}\right|\left|a_{3}-a_{2}^{2}\right| .
$$

Now, using Corollary 8, Theorems 9 and 10, and Lemma 11 , we get the required result. 


\section{Bounds of $H_{3,1}(f)$ for 2-Fold and 3-Fold Symmetric Functions}

Let $m \in \mathbb{N}=\{1,2,3, \cdots\}$, if a rotation of domain $\mathbb{D}$ about the origin through an angle $2 \pi / m$ carries itself on the domain $\mathbb{D}$ is called $m$-fold symmetric. It is very much clear to see that an analytic function $f$ is $m$-fold symmetric in $\mathbb{D}$, if

$$
f\left(e^{\frac{2 \pi}{m}} z\right)=e^{\frac{2 \pi}{m}} f(z), \quad z \in \mathbb{D}
$$

By $\mathcal{S}^{(m)}$, we mean the set of $m$-fold symmetric univalent functions having the following series form

$$
f(z)=z+\sum_{k=2}^{\infty} a_{\mathrm{m} k+1} z^{m k+1}, \quad z \in \mathbb{D} .
$$

The subclass $\mathcal{S}_{S G}^{*(m)}$ is a set of $m$-fold symmetric starlike functions associated with modified sigmoid function. More precisely, an analytic function $f$ of the form (57) belongs to class $\mathcal{S}_{S G}^{*(m)}$ if and only if

$$
\frac{z f^{\prime}(z)}{f(z)}=\frac{2}{1+e^{-((p(z)-1) /(p(z)+1))}}, \quad p \in \mathscr{P}^{(m)},
$$

where the set $\mathscr{P}^{(m)}$ is defined by

$$
\mathscr{P}^{(m)}=\left\{p \in \mathscr{P}: p(z)=1+\sum_{k=1}^{\infty} c_{m k} z^{m k}, \quad z \in \mathbb{D}\right\} .
$$

Theorem 13. If $f \in \mathcal{S}_{S G}^{*(2)}$ be of the form (57), then

$$
\left|H_{3,1}(f)\right| \leq \frac{1}{32}
$$

Proof. Since $f \in \mathcal{S}_{S G}^{*(2)}$; therefore, there exists a function $p \in$ $\mathscr{P}^{(2)}$ such that

$$
\frac{z f^{\prime}(z)}{f(z)}=\frac{2}{1+e^{-((p(z)-1) /(p(z)+1))}} .
$$

Using the series form (57) and (59), when $m=2$ in the above relation, we have

$$
\begin{aligned}
& a_{3}=\frac{1}{8} c_{2}, \\
& a_{5}=\frac{1}{16} c_{4}-\frac{3}{128} c_{2}^{2} .
\end{aligned}
$$

Now,

$$
H_{3,1}(f)=a_{3} a_{5}-a_{3}^{2}
$$

Therefore,

$$
H_{3,1}(f)=\frac{c_{2}}{128}\left(c_{4}-\frac{3}{8} c_{2}^{2}\right)
$$

Using (13) and (14) along with triangle inequality, we get

$$
\left|H_{3,1}(f)\right| \leq \frac{1}{32} \text {. }
$$

Theorem 14. If $f \in \mathcal{S}_{S G}^{*(3)}$ be of the form (57), then

$$
\left|H_{3,1}(f)\right| \leq \frac{1}{36}
$$

Proof. Since $f \in \mathcal{S}_{S G}^{*(3)}$; therefore, there exists a function $p \in$ $\mathscr{P}^{(3)}$ such that

$$
\frac{z f^{\prime}(z)}{f(z)}=\frac{2}{1+e^{-((p(z)-1) /(p(z)+1))}} .
$$

Using the series form (57) and (59), when $m=3$ in the above relation, we have

$$
a_{4}=\frac{1}{12} c_{3}
$$

Now,

$$
H_{3,1}(f)=-a_{4}^{2}
$$

Therefore,

$$
H_{3,1}(f)=\frac{1}{144} c_{3}^{2}
$$

Using (13), we get

$$
\left|H_{3,1}(f)\right| \leq \frac{1}{36}
$$

The result is best possible for function defined as follows:

$$
f_{4}(z)=z \exp \left(\int_{0}^{z} \frac{e^{t^{3}}-1}{t\left(e^{t^{3}}+1\right)} d t\right)=z+\frac{1}{6} z^{4}+\cdots
$$

\section{Data Availability}

The data used in this article are artificial and hypothetical, and anyone can use these data before prior permission by just citing this article.

\section{Conflicts of Interest}

The authors declare that they have no competing interests. 


\section{Authors' Contributions}

All authors jointly worked on the results, and they read and approved the final manuscript.

\section{References}

[1] L. De Branges, "A proof of the Bieberbach conjecture," Acta Mathematica, vol. 154, no. 1-2, pp. 137-152, 1985.

[2] S. S. Miller and P. T. Mocanu, "Second order differential inequalities in the complex plane," Journal of Mathematical Analysis and Applications, vol. 65, no. 2, pp. 289-305, 1978.

[3] S. S. Miller and P. T. Mocanu, "Differential subordinations," in Theory and Applications, Series on Monographs and Textbooks in Pure and Applied Mathematics. No. 225, pp. 101-105, Marcel Dekker Inc., New York, 2000.

[4] W. Janowski, "Extremal problems for a family of functions with positive real part and for some related families," Annales Polonici Mathematici, vol. 23, no. 2, pp. 159-177, 1970.

[5] M. S. Robertson, "Certain classes of starlike functions," The Michigan Mathematical Journal, vol. 32, pp. 135-140, 1985.

[6] F. Ronning, "Uniformly convex functions and a corresponding class of starlike functions," Proceedings of American Mathematical Society, vol. 118, no. 1, pp. 189-196, 1993.

[7] A. Jangteng, S. A. Halim, and M. Darus, "Coefficient inequality for starlike and convex functions," Int. J. Ineq. Math. Anal, vol. 1, pp. 619-625, 2007.

[8] J. Sokól, "Radius problems in the class," Applied Mathematics and Computation, vol. 214, no. 2, pp. 569-573, 2009.

[9] M. Raza and S. N. Malik, "Upper bound of the third hankel determinant for a class of analytic functions related with lemniscate of bernoulli," Journal of Inequalities and Applications, vol. 2013, no. 1, Article ID 412, 2013.

[10] A. Alotaibi, M. Arif, M. A. Alghamdi, and S. Hussain, "Starlikness associated with cosine hyperbolic function," Mathematics, vol. 8, no. 7, p. 1118, 2020.

[11] N. E. Cho, V. Kumar, S. Kumar, and V. Ravichandran, "Radius problems for starlike functions associated with the sine function," Bulletin of the Iranian Mathematical Society, vol. 45, no. 1, pp. 213-232, 2019.

[12] M. Arif, M. Raza, H. Tang, S. Hussain, and H. Khan, "Hankel determinant of order three for familiar subsets of analytic functions related with sine function," Open Mathematics, vol. 17, no. 1, pp. 1615-1630, 2019.

[13] R. K. Raina and J. Sokół, "On coefficient estimates for a class of starlike functions," Hacettepe Journal of Mathematics and Statistics, vol. 44, pp. 1427-1433, 2015.

[14] R. Mendiratta, S. Nagpal, and V. Ravichandran, "On a subclass of strongly starlike functions associated with exponential function," Bulletin of the Malaysian Mathematical Sciences Society, vol. 38, pp. 365-386, 2015.

[15] L. Shi, H. M. Srivastava, M. Arif, S. Hussain, and H. Khan, "An investigation of the third hankel determinant problem for certain subfamilies of univalent functions involving the exponential function," Symmetry, vol. 11, no. 5, p. 598, 2019.

[16] K. Sharma, N. K. Jain, and V. Ravichandran, "Starlike functions associated with a cardioid," Afrika Matematika, vol. 27, no. 5-6, pp. 923-939, 2016.

[17] L. Shi, A. Izaz, M. Arif, N. E. Cho, and S. Hussain, "A study of third Hankel determinant problem for certain subfamilies of analytic functions involving cardioid domain," Mathematics, vol. 7, no. 5, p. 418, 2019.

[18] K. Bano and M. Raza, "Starlike functions associated with cosine functions," Bulletin of the Iranian Mathematical Society, 2020.

[19] N. E. Cho, S. Kumar, V. Kumar, V. Ravichandran, and H. M. Serivasatava, "Starlike functions related to the Bell numbers," Symmetry, vol. 11, no. 2, p. 219, 2019.

[20] J. Dzoik, R. K. Raina, and J. Sokół, “On a class of starlike functions related to a shell-like curve connected with Fibonacci numbers," Mathematical and Computer Modelling, vol. 57, no. 5-6, pp. 1203-1211, 2013.

[21] S. Kanas and D. Răducanu, "Some class of analytic functions related to conic domains," Mathematica slovaca, vol. 64, pp. 1183-1196, 2014.

[22] S. Kumar and V. Ravichandran, "A subclass starlike functions associated with rational function," Southeast Asian Bulletin of Mathematics, vol. 40, pp. 199-212, 2016.

[23] O. A. Fadipe-Joseph, A. T. Oladdipo, and A. U. Ezeafulukwe, "Modified sigmoid function in univalent function theory," International Journal of Mathematical Sciences and Engineering Applications, vol. 7, pp. 313-317, 2013.

[24] P. Goel and S. Kumar, "Certain class of starlike functions associated with modified sigmoid function," Bulletin of the Malaysian Mathematical Sciences Society, vol. 43, pp. 957-991, 2019.

[25] A. Jangteng, S. A. Halim, and M. Darus, "On Hankel determinants of univalent functions," Mathematika, vol. 14, pp. 108$112,1967$.

[26] A. Jangteng, S. A. Halim, and M. Darus, "On the coefficient and Hankel determinants of univalent functions. Inequality for starlike and convex functions," Journal of the London Mathematical Society, vol. 1, pp. 111-122, 1966.

[27] A. Jangteng, S. A. Halim, and M. Darus, "Coefficient inequality for a function whose derivative has a positive real part," Journal of Inequalities in Pure and Applied Mathematics, vol. 7, pp. 1-5, 2006.

[28] D. Răducanu and P. Zaprawa, "Second Hankel determinant for close-to-convex functions," Comptes Rendus Mathematique, vol. 355, no. 10, pp. 1063-1071, 2017.

[29] D. V. Krishna and T. Ram Reddy, "Second Hankel determinant for the class of Bazilevic functions," Studia Universitatis Babeș-Bolyai Mathematica, vol. 60, pp. 413-420, 2015.

[30] D. Bansal, "Upper bound of second Hankel determinant for a new class of analytic functions," Applied Mathematics Letters, vol. 23, pp. 103-107, 2013.

[31] S. K. Lee, V. Ravichandran, and S. Supramaniam, "Bounds for the second Hankel determinant of certain univalent functions," Journal of inequalities and Applications, vol. 2013, no. $1,2013$.

[32] M. S. Liu, J. F. Xu, and M. Yang, "Upper bound of second Hankel determinant for certain subclasses of analytic functions," Abstract and Applied Analysis, vol. 2014, Article ID 603180, 10 pages, 2014.

[33] J. W. Noonan and D. K. Thomas, "On the second Hankel determinant of areally mean p-valent functions," Transactions of the American Mathematical Society, vol. 223, pp. 337-346, 1976.

[34] H. Orhan, N. Magesh, and J. Yamini, "Bounds for the second Hankel determinant of certain bi-univalent functions," Turkish Journal of Mathematics, vol. 40, pp. 679-687, 2016. 
[35] K. O. Babalola, "On $\mathrm{H}_{3}$ (1) Hankel determinant for some classes of univalent functions," Inequality Theory and Applications, vol. 6, pp. 1-7, 2010.

[36] S. Altinkaya and S. Yalçin, "Third Hankel determinant for Bazilevic functions," Advances in Math, vol. 5, pp. 91-96, 2016.

[37] M. Arif, K. N. Noor, and M. Raza, "Hankel determinant problem of a subclass of analytic functions," Journal of Inequalities and Applications, vol. 2012, no. 1, pp. 1-7, 2012.

[38] D. Bansal, S. Maharana, and J. K. Prajapat, "Third order Hankel determinant for certain univalent functions," Journal of the Korean Mathematical Society, vol. 52, no. 6, pp. 1139-1148, 2015.

[39] N. E. Cho, B. Kowalczyk, O. S. Kwon, A. Lecko, and J. Sim, "Some coefficient inequalities related to the Hankel determinant for strongly starlike functions of order alpha," Journal of Mathematical Inequalities, vol. 11, pp. 429-439, 2017.

[40] D. V. Krishna, B. Venkateswarlua, and T. RamReddy, “Third Hankel determinant for bounded turning functions of order alpha," Journal of the Nigerian Mathematical Society, vol. 34, no. 2, pp. 121-127, 2015.

[41] G. Shanmugam, B. A. Stephen, and K. O. Babalola, "Third Hankel determinant for $\alpha$-starlike functions," Gulf Journal of Mathematics, vol. 2, pp. 107-113, 2014.

[42] P. Zaprawa, "Third Hankel determinants for subclasses of univalent functions," Mediterranean Journal of Mathematics, vol. 14, no. 1, p. 19, 2017.

[43] B. Kowalczyk, A. Lecko, and Y. J. Sim, "The sharp bound for the Hankel determinant of the third kind for convex functions," Bulletin of the Australian Mathematical Society, vol. 97, no. 3, pp. 435-445, 2018.

[44] A. Lecko, Y. J. Sim, and B. S'miarowska, "The sharp bound of the Hankel determinant of the third kind for starlike functions of order 1/2," Complex Analysis and Operator Theory, vol. 13, no. 5, pp. 2231-2238, 2019.

[45] O. S. Kwon, A. Lecko, and Y. J. Sim, "The bound of the Hankel determinant of the third kind for starlike functions," Bulletin of the Malaysian Mathematical Sciences Society, vol. 42, pp. 1-14, 2018.

[46] F. Keogh and E. Merkes, "A coefficient inequality for certain classes of analytic functions," Proceedings of the American Mathematical Society, vol. 20, no. 1, pp. 8-12, 1969.

[47] C. Pommerenke, Univalent Functions, Math, Lehrbucher, vandenhoeck and Ruprecht, Gottingen, 1975.

[48] W. Ma and M. Minda, "A unified treatment of some special classes of univalent functions," Proceedings of the Conference on Complex Analysis, Z. Li, F. Ren, L. Yang, and S. Zhang, Eds., , pp. 157-169, International Press, Cambridge, MA, USA, 1992. 